\title{
HEAT CONDUCTION IN HOLLOW \\ CYLINDERS WITH RADIATION
}

\author{
by E. MARCHI and G. ZGRABLICH \\ (Received 20th January 1964)
}

\begin{abstract}
A new finite integral transformation (an extension of those given by Sneddon (1)), whose kernel is given by cylindrical functions, is used to solve the problem of finding the temperature at any point of a hollow cylinder of any height, with boundary conditions of radiation type on the outside and inside surfaces, with independent radiation constants. It is to be noticed that all possible problems on boundary conditions in hollow cylinders can be solved by particularising the method described here.
\end{abstract}

\section{Introduction}

Our purpose is to solve the problem of finding the temperature at any point of a hollow cylinder of any height, when there is heat radiation on its outside and inside surfaces. We suppose that the media bounding the two cylindrical surfaces are, in general, different. For that purpose, we introduce, in the first part, a new finite integral transform, restricting our attention to the property necessary fcr the solution of the problem that will be stated and solved in the second part. We assume that all functions involved satisfy Dirichlet's conditions in the intervals considered.

\section{The transformation and its essential property}

Let us seek the solution of the Bessel differential equation of order $p$

$$
\frac{d^{2} y}{d x^{2}}+\frac{1}{x} \frac{d y}{d x}+\left(\mu^{2}-p^{2} / x^{2}\right) y=0
$$

for the boundary conditions

$$
y(a)+k_{1} y^{\prime}(a)=0 ; y(b)+k_{2} y^{\prime}(b)=0
$$

where $a<b$. If the general solution of the equation (1) is

$$
y(x)=C_{1} J_{p}(\mu x)+C_{2} G_{p}(\mu x)
$$

where $J_{p}(\mu x)$ and $G_{p}(\mu x)=\frac{1}{2} \pi \operatorname{cosec}(p \pi)\left[J_{-p}(\mu x)-e^{-i p \pi} J_{p}(\mu x)\right]$ are Bessel functions of first and second kind, respectively, of order $p$, then by substitution 
of (3) into (2) we obtain

where

$$
C_{1} J_{p}\left(k_{1}, \mu a\right)=-C_{2} G_{p}\left(k_{1}, \mu a\right), C_{1} J_{p}\left(k_{2}, \mu b\right)=-C_{2} G_{p}\left(k_{2}, \mu b\right)
$$

$J_{p}\left(k_{i}, \mu x\right)=J_{p}(\mu x)+k_{i} \mu J_{p}^{\prime}(\mu x), G_{p}\left(k_{i}, \mu x\right)=G_{p}(\mu x)+k_{i} \mu G_{p}^{\prime}(\mu x) \quad(i=1,2)$.

From equation (4), we see that solutions exist only if:

$$
J_{p}\left(k_{1}, \mu a\right) G_{p}\left(k_{2}, \mu b\right)-J_{p}\left(k_{2}, \mu b\right) G_{p}\left(k_{1}, \mu a\right)=0 .
$$

Denote by $\mu_{n}$ the positive roots of this equation. From the first equation of (4) and (3) we obtain:

$$
y_{n}(x)=C_{1} / G_{p}\left(k_{1}, \mu_{n} a\right)\left[J_{p}\left(\mu_{n} x\right) G_{p}\left(k_{1}, \mu_{n} a\right)-G_{p}\left(\mu_{n} x\right) J_{p}\left(k_{1}, \mu_{n} a\right)\right] .
$$

On the other hand, by using the second equation of (4) and (3) we find

$$
y_{n}(x)=C_{1} / G_{p}\left(k_{2}, \mu_{n} b\right)\left[J_{p}\left(\mu_{n} x\right) G_{p}\left(k_{2}, \mu_{n} b\right)-G_{p}\left(\mu_{n} x\right) J_{p}\left(k_{2}, \mu_{n} b\right)\right] .
$$

Linear combination of the two preceding equations leads to the functions

$$
\begin{aligned}
S_{p}\left(k_{1}, k_{2}, \mu_{n} x\right)=J_{p}\left(\mu_{n} x\right)\left[G_{p}\left(k_{1}, \mu_{n} a\right)\right. & \left.+G_{p}\left(k_{2}, \mu_{n} b\right)\right] \\
& -G_{p}\left(\mu_{n} x\right)\left[J_{p}\left(k_{1}, \mu_{n} a\right)+J_{p}\left(k_{2}, \mu_{n} b\right)\right]
\end{aligned}
$$

which are solutions of Bessel's differential equation of order $p$ and satisfy the boundary conditions (2). Because of this fact, such functions are orthogonal in the interval $(a, b)$. Now, let us define the finite integral transform

$$
f(n)=\int_{a}^{b} x f(x) S_{p}\left(k_{1}, k_{2}, \mu_{n} x\right) d x .
$$

As is well known (1), the inversion theorem is given by

$$
f(x)=\sum_{n} a_{n} S_{p}\left(k_{1}, k_{2}, \mu_{n} x\right)
$$

where the sum must be taken over the positive roots of equation (6). From the orthogonality of the functions defined by (7), the $a_{n}$ are given by

where:

$$
a_{n}=\vec{f}_{p}(n) / C_{n}
$$

By using the relation $\dagger$

$$
C_{n}=\int_{a}^{b} x\left[S_{p}\left(k_{1}, k_{2}, \mu_{n} x\right)\right]^{2} d x
$$

$$
\begin{aligned}
\int^{z} z \mathscr{C}_{\mu}(k z) \overline{\mathscr{C}}_{\mu}(k z) d z=\frac{1}{4} z^{2}\left\{2 \mathscr{C}_{\mu}(k z) \overline{\mathscr{C}}_{\mu}(k z)-\mathscr{C}_{\mu-1}(k z) \overline{\mathscr{C}}_{\mu+1}(k z)\right. \\
\left.-\mathscr{C}_{\mu+1}(k z) \overline{\mathscr{C}}_{\mu-1}(k z)\right\}
\end{aligned}
$$

where $\mathscr{C}_{\mu}(k z)$ and $\overline{\mathscr{C}}_{\mu}(k z)$ are cylindrical functions of order $p$, there results:

$$
\begin{gathered}
C_{n}=\frac{1}{2} b^{2}\left\{\tilde{S}_{p}^{2}\left(\left(k_{1}, k_{2}, \mu_{n} b\right)-\tilde{S}_{p-1}\left(k_{1}, k_{2}, \mu_{n} b\right) \tilde{S}_{p+1}\left(k_{1}, k_{2}, \mu_{n} b\right)\right\}\right. \\
-\frac{1}{2} a^{2}\left\{\tilde{S}_{p}^{2}\left(k_{1}, k_{2}, \mu_{n} a\right)-\tilde{S}_{p-1}\left(k_{1}, k_{2}, \mu_{n} a\right) \tilde{S}_{p+1}\left(k_{1}, k_{2}, \mu_{n} a\right)\right\}, \\
\dagger \operatorname{See}(2), \text { p. } 134 .
\end{gathered}
$$


where

$$
\begin{aligned}
\tilde{S}_{q}\left(k_{1}, k_{2}, \mu_{n} x\right)=J_{q}\left(\mu_{n} x\right)\left[G_{p}\left(k_{1}, \mu_{n} a\right)+G_{p}\left(k_{2}, \mu_{n} b\right)\right] & \\
& -G_{q}\left(\mu_{n} x\right)\left[J_{p}\left(k_{1}, \mu_{n} a\right)+J_{p}\left(k_{2}, \mu_{n} b\right)\right]
\end{aligned}
$$

To solve the problem stated above, it is sufficient to find only the effect of the transformation on the expression $\frac{d^{2} f}{d x^{2}}+\frac{1}{x} \frac{d f}{d x}-\frac{p^{2}}{x^{2}} f$. By integrating by parts, we obtain:

$$
\begin{aligned}
\int_{a}^{b} x\left(\frac{d^{2} f}{d x^{2}}+\frac{1}{x} \frac{d f}{d x}\right) S_{p}\left(k_{1}, k_{2}, \mu_{n} x\right) d x \\
=\left[x S_{p}\left(k_{1}, k_{2}, \mu_{n} x\right)\left\{\frac{d f}{d x}-\mu_{n} S_{p}^{\prime}\left(k_{1}, k_{2}, \mu_{n} x\right) / S_{p}\left(k_{1}, k_{2}, \mu_{n} x\right) f\right\}\right]_{a}^{b} \\
\quad+\mu_{n}^{2} \int_{a}^{b} x f \frac{d^{2}}{d x^{2}} S_{p}\left(k_{1}, k_{2}, \mu_{n} x\right) d x+\mu_{n} \int_{a}^{b} f \frac{d}{d x} S_{p}\left(k_{1}, k_{2}, \mu_{n} x\right) d x \ldots
\end{aligned}
$$

On the other hand, from (7) and (5), and taking into account (6), we have by direct calculation

$$
\begin{aligned}
& S_{p}^{\prime}\left(k_{1}, k_{2}, \mu_{n} a\right) / S_{p}\left(k_{1}, k_{2}, \mu_{n} a\right)=-1 / k_{1} \mu_{n} ; \\
& S_{p}^{\prime}\left(k_{1}, k_{2}, \mu_{n} b\right) / S_{p}\left(k_{1}, k_{2}, \mu_{n} b\right)=-1 / k_{2} \mu_{n} .
\end{aligned}
$$

By substituting (13) into (12), and remembering that the functions $S_{p}\left(k_{1}, k_{2}, \mu_{n} x\right)$ satisfy Bessel's differential equation of order $p$, we obtain finally:

$$
\begin{aligned}
\int_{a}^{b} x\left(\frac{d^{2} f}{d x^{2}}+\frac{1}{x} \frac{d f}{d x}-\right. & \left.\frac{p^{2}}{x^{2}} f\right) S_{p}\left(k_{1}, k_{2}, \mu_{n} x\right) d x \\
& =b / k_{2} S_{p}\left(k_{1}, k_{2}, \mu_{n} b\right)\left[f+k_{2} \frac{d f}{d x}\right]_{x=b} \\
& -a / k_{1} S_{p}\left(k_{1}, k_{2}, \mu_{n} a\right)\left[f+k_{1} \frac{d f}{d x}\right]_{x=a}-\mu_{n}^{2} f_{p}(n) .
\end{aligned}
$$

This is the basic property of our transform, that will enable us to solve the problem which we now state.

\section{Application to the hollow cylinder with radiation}

Consider the hollow cylinder whose axis is coincident with the $z$ axis, defined by $0 \leqq z \leqq h$ and $a \leqq r \leqq b$, where $a$ and $b$ are the external and internal radii, respectively, and $(r, \phi, z)$ are cylindrical coordinates. Let us consider the heat conduction problem, with symmetry with respect to the $z$ axis. Then the temperature $\theta(r, z, t)$ at any point of the cylinder, where $t$ is the time, will be the solution of the conduction equation

$$
\frac{\partial^{2}}{\partial r^{2}} \theta(r, z, t)+\frac{1}{r} \frac{\partial}{\partial r} \theta(r, z, t)+\frac{\partial^{2}}{\partial z^{2}} \theta(r, z, t)=\frac{1}{\mathscr{H}} \frac{\partial}{\partial t} \theta(r, z, t)
$$


where $\mathscr{H}=K / \rho c$, where $K$ is the conductibility of the medium, $\rho$ is its density and $c$ its calorific capacity, assumed to be constant, with boundary and initial conditions

$$
\begin{aligned}
& \theta(a, z, t)+k_{1} \frac{\partial}{\partial r} \theta(a, z, t)=F_{a}(z, t) \text { for all } 0<z<h \text { and } t>0, \\
& \theta(b, z, t)+k_{2} \frac{\partial}{\partial r} \theta(b, z, t)=F_{b}(z, t) \text { for all } 0<z<h \text { and } t>0,
\end{aligned}
$$

where $k_{1}$ and $k_{2}$ are the radiation constants on the two cylindrical surfaces,

$$
\begin{array}{ll}
\theta(r, h, t)=0 & \text { for all } a<r<b \text { and } t>0, \\
\theta(r, 0, t)=0 & \text { for all } a<r<b \text { and } t>0, \\
\theta(r, z, 0)=\theta_{0}(r, z) & \text { for all } a<r<b \text { and } 0<z<h,
\end{array}
$$

where $F_{a}(z, t), F_{b}(z, t)$ and $\theta_{0}(r, z)$ are known. By application to (15) of the transformation defined in (8), with respect to $r$, with $p=0$, writing

$$
\bar{\theta}(n, z, t)=\int_{a}^{b} r \theta(r, z, t) S_{0}\left(k_{1}, k_{2}, \mu_{n} r\right) d r
$$

where $\mu_{n}$ are positive roots of equation (6) with $p=0$, and taking into account (16), we obtain

$$
\mathscr{H} \frac{\partial^{2}}{\partial z^{2}} \bar{\theta}(n, z, t)-\frac{\partial}{\partial t} \bar{\theta}(n, z, t)-\mu_{n}^{2} \mathscr{H} \bar{\theta}(n, z, t)=\mathscr{H} \chi(z, t)
$$

where

$$
\chi(z, t)=a / k_{1} S_{0}\left(k_{1}, k_{2}, \mu_{n} a\right) F_{a}(z, t)-b / k_{2} S_{0}\left(k_{1}, k_{2}, \mu_{n} b\right) F_{b}(z, t)
$$

is obviously a known function. To solve the differential equation (19), let us introduce the Fourier transform, in the variable $z$ :

$$
\overline{\bar{\theta}}(n, m, t)=\int_{0}^{h} \bar{\theta}(n, z, t) \sin (m \pi z / h) d z
$$

From this, and the property $\uparrow$ :

$$
\begin{aligned}
\int_{0}^{h} \frac{\partial^{2}}{\partial z^{2}} \bar{\theta}(n, z, t) \sin ( & m \pi z / h) d z \\
& =m \pi / h\left[(-1)^{m+1} \bar{\theta}(n, h, t)+\bar{\theta}(n, 0, t)\right]-m^{2} \pi^{2} / h^{2} \overline{\bar{\theta}}(n, m, t)
\end{aligned}
$$

and remembering (17), (19) is transformed into:

where

$$
d / d t \overline{\bar{\theta}}(n, m, t)+\mathscr{H}\left(\mu_{n}^{2}+m^{2} \pi^{2} / h^{2}\right) \overline{\bar{\theta}}(n, m, t)=-\mathscr{H} \chi_{s}(m, t)
$$

$$
\chi_{s}(m, t)=\int_{0}^{h} \chi(z, t) \sin (m \pi z / h) d z
$$

By introducing the Laplace transform $L[\psi(t)]=\int_{0}^{\infty} e^{-p t} \psi(t) d t$ and taking $\dagger$ See (1), p. 75. 
into account the initial condition (18), (21) is transformed into

$$
\begin{aligned}
L[\overline{\bar{\theta}}(n, m, t)]=\overline{\bar{\theta}}_{0}(n, m) / p+\mathscr{H}\left(\mu_{n}^{2}+m^{2} \pi^{2} / h^{2}\right) \\
-\mathscr{H} L\left[\chi_{s}(m, t)\right] / p+\mathscr{H}\left(\mu^{2}+m^{2} \pi^{2} / h^{2}\right) .
\end{aligned}
$$

Now, if we apply the operator $L^{-1}$ to (22), and remember the convolution theorem for Laplace transforms, we obtain

$$
\begin{aligned}
\bar{\theta}(n, m, t)=\bar{\theta}_{0}(n, m) \exp \left[-\mathscr{H}\left(\mu_{n}^{2}+m^{2} \pi^{2} / h^{2}\right) t\right] \\
-\mathscr{H} \int_{0}^{t} \chi_{s}(m, u) \exp \left[-\mathscr{H}\left(\mu_{n}^{2}+m^{2} \pi^{2} / h^{2}\right)(t-u)\right] d u .
\end{aligned}
$$

Using, now, the inversion theorem for our Fourier transform, there results

$$
\bar{\theta}(n, z, t)=2 / h \sum_{m=1}^{\infty} \overline{\bar{\theta}}(n, m, t) \sin (m \pi z / h)
$$

and finally, by the inversion theorem (9), and by substitution of (23) into (24), we have:

$$
\begin{aligned}
& \theta(r, z, t)=2 / h \sum_{n} \sum_{m} 1 / C_{n}\left\{\bar{\theta}_{0}(n, m) \exp \left[-\mathscr{H}\left(\mu_{n}^{2}+m^{2} \pi^{2} / h^{2}\right) t\right]\right. \\
& \left.-\mathscr{H} \int_{0}^{t} \chi_{s}(m, u) \exp \left[-\mathscr{H}\left(\mu_{n}^{2}+m^{2} \pi^{2} / h^{2}\right)(t-u)\right] d u\right\} \cdot \sin (m \pi z / h) S_{0}\left(k_{1}, k_{2}, \mu_{n} r\right)
\end{aligned}
$$

where $C_{n}$ are given by (11) with $p=0$.

Remarks. We have chosen for solution a particular problem of the type that can be solved with this new transform. This has been done to show the specific application of our transformation when there is cylindrical symmetry with radiation in both cylindrical surfaces and, in general, with different bounding media. For this reason, and to simplify the calculations, we have taken the very particular conditions (17). The problem can however be solved with these conditions expressed in a more general form. As will be noticed, the problem is completely general, and all the cases can be obtained by assigning arbitrary values to $k_{1}$ and $k_{2}$. For example, if $k_{1}=k_{2}=0$, the problem for a specified temperature on the boundary can be solved with only slight modifications. On the other hand, if $k_{1}$ and $k_{2}$ tend to infinity, there results the problem with boundary conditions:

$$
\frac{\partial}{\partial r} \theta(a, z, t)=\frac{\partial}{\partial r} \theta(b, z, t)=0 .
$$

Other cases of interest are those obtained when $k_{1}=0, k_{2} \neq 0$ or $k_{1} \neq 0$, $k_{2}=0$ or $k_{1} \rightarrow \infty, k_{2}=0$, etc. Moreover, each of these cases can be treated, without difficulty, for $h \rightarrow \infty$, using an infinite, instead of a finite, Fourier transform. 


\section{REFERENCES}

(1) I. SNEDDON, Fourier Transforms (McGraw-Hill, 1951).

(2) G. N. Watson, A Treatise on the Theory of Bessel Functions (Macmillan, 1948).

Facultad de Ciencias, U.N.C.

SAN Luis, Argentina 